\title{
Goldberger-Treiman constraint criterion for hyperon coupling constants
}

\author{
Ignacio J. General and Stephen R. Cotanch \\ Department of Physics, North Carolina State University, Raleigh, North Carolina 27695-8202
}

(Dated: December 22, 2018)

\begin{abstract}
The generalized Goldberger-Treiman relation is combined with the Dashen-Weinstein sum rule to provide a constraint equation between the $g_{K \Sigma N}$ and $g_{K \Lambda N}$ coupling constants. A comprehensive examination of the published phenomenological and theoretical hyperon couplings has yielded a much smaller set of values, spanning the intervals $0.80 \leq g_{K \Sigma N} / \sqrt{4 \pi} \leq 2.72$ and $-3.90 \leq g_{K \Lambda N} / \sqrt{4 \pi} \leq-1.84$, consistent with this criterion. The $S U_{F}(3)$ and Goldberger-Treiman hyperon couplings satisfy the constraint along with predictions from a Taylor series extrapolation using the same momentum variation as exhibited by $g_{\pi N N}$.
\end{abstract}

PACS numbers: 11.30.Hv, 11.40.Ha, 13.30.Ce, 13.75.Gx, 13.75.Jz

\section{INTRODUCTION}

After a half century of investigating meson-baryon interactions, it is somewhat surprising that there are still several important coupling constants not accurately known. While the $\pi N$ coupling constant, $g_{\pi N N}$, has been determined to within a few percent, significant uncertainty in the two hyperon couplings, $g_{K(Y=\Lambda, \Sigma) N}$, remains and even recently published values for both vary by more than a factor of four. This large variance is due to limited experimental information and also shortcomings in theoretical models. Further, analyses of purely hadronic processes typically yield larger couplings than those obtained from hyperon electromagnetic production studies. Fortunately, with the advent of new accelerator facilities, such as Jefferson Lab and SPring-8, more accurate and abundant data are now becoming available. Related, the recently reported [1] discovery of the exotic strangeness +1 pentaquark resonance, $\Theta^{+}$, is also attracting attention which should spawn additional $K N$ measurements.

The purpose of this work is to detail a potentially useful constraint relation between $g_{K \Lambda N}$ and $g_{K \Sigma N}$ which should facilitate future hyperon scattering and production analyses, especially with respect to extracting more accurate coupling constants. The constraint involves the generalized Goldberger-Tremain (GT) relation 2] and the Dashen-Weinstein (DW) sum-rule [3]. The GT relation is exact in the combined chiral and zero momentum limits according to the partially conserved axial vector current (PCAC) hypothesis and the assumed slow momentum variation of the $\pi N$ coupling constant. Even with explicit chiral symmetry breaking, the nucleon GT relation remains valid and is now satisfied to within one percent (see section II). Because of the larger strange quark mass and attending broken $S U_{F}(3)$ flavor symmetry, the generalized GT relation is not as accurate in the hyperon sector. However the deviation, or hyperon GT discrepancy, $\Delta_{Y}$, is believed to be reasonably accurately constrained by the DW sum rule since corrections are suppressed by two powers in the heavy baryon chiral perturbation theory expansion [4]. Accordingly, by utilizing the DW sum rule connecting the $N, \Lambda$ and $\Sigma$ dis- crepancies, we have obtained a presumably accurate constraint equation between the hyperon and nucleon coupling constants, involving the known axial charges and hadron masses.

There have been several studies 4, 5, 6, 7] that have implemented the DW sum rule, especially to constrain the $\pi N$ coupling constant [4, 5, 6]. Our approach represents a different view as we submit the hyperon couplings and their discrepancies are the limiting, less accurate quantities and that the reasonably well known $g_{\pi N N}$, through the DW sum rule, provides a constraint for $g_{K \Lambda N}$ and $g_{K \Sigma N}$. Because $g_{\pi N N}$ is only determined to within a few percent, the constraint equation produces a band in the $\left(g_{K \Sigma N}, g_{K \Lambda N}\right)$ plane and we document which published couplings, when plotted, fall within this band. Since there is only one equation for the two couplings our constraint will only be useful for analyses involving both couplings. However, this should encompass most phenomenological investigations since models for hyperon reactions and production entail both $\Lambda$ and $\Sigma$ intermediate states and their attending couplings. Because of this interdependence our criterion should be useful even if analyses of purely hadronic processes continue to provide larger hyperon couplings than electromagnetic production (i.e. the constraint should provide a good numerical relation between the two couplings even if there is an effective coupling renormalization due to model dependence). Our result should also be of special interest to the hyperon and hypernuclear community and of timely benefit in the analysis of precision kaon electromagnetic production data recently measured 8 at Jefferson Lab. Obtaining improved hyperon coupling constants will also permit new confrontations with QCD based theoretical approaches which have been successful in calculating $g_{\pi N N}[9]$.

This paper is organized into four sections. The generalized GT relation and the DW sum rule, along with the $S U_{F}(3)$ coupling relations, are given in section II and the constraint criterion is developed. Published values for $g_{K Y N}$ are reviewed in section III and the coupling constraint is imposed producing a subset satisfying this criterion. Finally, conclusions are summarized in section IV. 


\section{FUNDAMENTAL COUPLING RELATIONS}

\section{A. Generalized Goldberger-Treiman relation}

To fully appreciate the validity of the GT relation it is illustrative to sketch its derivation. There are several ways to obtain this result such as using PCAC or unsubtracted dispersion relations (pion pole dominance of the axial vector divergence). Here the PCAC approach is adopted. Consider the matrix element of the axial $S U_{F}(3)$ current operator, $j_{a}^{\mu 5}(x)=\bar{\Psi}(x) \gamma_{\mu} \gamma_{5} \frac{\lambda^{a}}{2} \Psi(x)$, between two baryon octet states, $\left\langle B\left|j_{a}^{\mu 5}\right| B^{\prime}\right\rangle$. Since the current transforms as a pseudovector, the most general form for this matrix element is

$$
\begin{aligned}
& \left\langle B\left|j_{a}^{\mu 5}(x)\right| B^{\prime}\right\rangle=e^{-i q \cdot x} \bar{u}(p)\left[g_{A}^{B}\left(q^{2}\right) \gamma^{\mu} \gamma_{5}\right. \\
& \left.+g_{T}^{B}\left(q^{2}\right) i \sigma^{\mu \nu} q_{\nu} \gamma_{5}+g_{P}^{B}\left(q^{2}\right) q^{\mu} \gamma_{5}\right] u\left(p^{\prime}\right)
\end{aligned}
$$

where $q=p-p^{\prime}, p^{2}=m_{B}^{2},{p^{\prime}}^{2}=m_{B^{\prime}}^{2}$ and $g_{A}^{B}\left(q^{2}\right)$ and $g_{P}^{B}\left(q^{2}\right)$ are the axial vector and induced pseudoscalar form factors, respectively. The induced tensor form factor, $g_{T}^{B}\left(q^{2}\right)$, violates $G$-parity and will be omitted, consistent with small effects from second class currents. The axial current operator also appears in the definition of the decay constant, $f_{M}$, for a pseudoscalar octet meson $M^{b}$ having mass $m_{M}$

$$
\left\langle 0\left|j_{a}^{\mu 5}(x)\right| M^{b}(q)\right\rangle=i \sqrt{2} f_{M} q^{\mu} \delta_{a}^{b} e^{-i q \cdot x} .
$$

Taking the divergence

$$
\left\langle 0\left|\nabla_{\mu} j_{a}^{\mu 5}(x)\right| M^{b}(q)\right\rangle=\sqrt{2} f_{M} m_{M}^{2} \delta_{a}^{b} e^{-i q \cdot x},
$$

yields a conserved axial current in the generalized chiral limit $\left(\lim _{m_{M} \rightarrow 0} \nabla_{\mu} j_{a}^{\mu 5}=0\right.$ ); this is the PCAC hypothesis. Under this assumption the baryon axial vector current, Eq. (1), is also conserved, and its divergence yields

$$
\bar{u}(p)\left[g_{A}^{B}\left(q^{2}\right) \not q+g_{P}^{B} q^{2}\right] \gamma_{5} u\left(p^{\prime}\right)=0 .
$$

Then using the free Dirac equation for the first term leads to the form factor relation

$$
g_{A}^{B}\left(q^{2}\right)=-\frac{q^{2}}{m_{B}+m_{B^{\prime}}} g_{P}^{B}\left(q^{2}\right) .
$$

To proceed further, consider the leading Feynman diagrams for the weak decay $B \rightarrow B^{\prime}+\bar{\nu}_{l}+l$ depicted in Fig. 1. Only the meson exchange graph c) contributes to $g_{P}^{B}$. Direct evaluation gives

$$
g_{P}^{B}\left(q^{2}\right)=-\frac{\sqrt{2} f_{M}}{q^{2}-m_{M}^{2}} g_{M B B^{\prime}},
$$

where $g_{M B B^{\prime}}$ is the strong interaction baryon-meson coupling constant. Including higher order vertex corrections would modify this result by an additional multiplicative form factor, $F\left(q^{2}\right)$, with $F(0)=1$. Combining Eqs. (56)
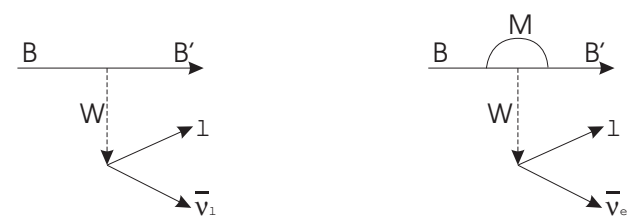

a) baryon decay

b) meson vertex correction

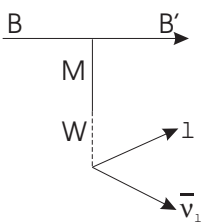

c) meson exchange

FIG. 1: Baryon weak decay and meson corrections.

finally yields in the combined chiral and zero momentum limits

$$
g_{A}^{B}(0)=\frac{\sqrt{2} f_{M}}{m_{B}+m_{B^{\prime}}} g_{M B B^{\prime}}(0),
$$

or rearranging

$$
g_{M B B^{\prime}}^{G T} \equiv g_{M B B^{\prime}}(0)=\frac{\left(m_{B}+m_{B^{\prime}}\right)}{\sqrt{2} f_{M}} g_{A}^{B}(0) \text {. }
$$

This is the generalized GT relation that defines the GT coupling constant and is exact in the zero meson mass and momentum limits, $m_{M}^{2}=q^{2}=0$.

We now apply Eq. (8) to evaluate the GT coupling constants $g_{\pi N N}^{G T}$ and $g_{K Y N}^{G T}$. First we specify our coupling constant convention and phase consistent with the usual pseudoscalar Lagrangian

$$
\begin{array}{r}
\mathcal{L}=i g_{\pi N N} \bar{N} \gamma_{5} \boldsymbol{\tau} N \cdot \boldsymbol{\pi}+i g_{K \Lambda N} \bar{N} \gamma_{5} \Lambda K \\
+i g_{K \Sigma N} \bar{N} \gamma_{5} \boldsymbol{\tau} \cdot \boldsymbol{\Sigma} K+\text { h.c. }
\end{array}
$$

with isospin nucleon, $N=\left(\begin{array}{l}p \\ n\end{array}\right)$, and kaon, $K=\left(\begin{array}{c}K^{+} \\ K^{0}\end{array}\right)$, doublet, and pion, $\boldsymbol{\pi}$, and sigma, $\boldsymbol{\Sigma}$, triplet fields. The different meson charge couplings are related to the generic coupling constants by

$$
\begin{aligned}
& g_{\pi N N} \equiv g_{\pi^{0} n n}=-\frac{1}{\sqrt{2}} g_{\pi^{-} n p}, \\
& g_{K \Lambda N} \equiv g_{K^{-} \Lambda p}, \\
& g_{K \Sigma N} \equiv \frac{1}{\sqrt{2}} g_{K^{-\Sigma^{-}} n} .
\end{aligned}
$$

With this notation and the most recently measured parameters [10] listed in Table【 including the axial charges (note $g_{A}^{N}=-g_{A}^{n}$ ) corresponding to the weak decays $n \rightarrow p+e^{-}+\bar{\nu}_{e}, \Lambda \rightarrow p+e^{-}+\bar{\nu}_{e}$ and $\Sigma^{-} \rightarrow n+e^{-}+\bar{\nu}_{e}$, 
the GT coupling constants are

$$
\begin{aligned}
g_{\pi N N}^{G T} & =\frac{g_{A}^{N}}{f_{\pi}} \frac{\left(m_{n}+m_{p}\right)}{2}=12.897 \pm 0.047, \\
g_{K \Lambda N}^{G T} & =\frac{g_{A}^{\Lambda}}{\sqrt{2} f_{K}}\left(m_{\Lambda}+m_{p}\right)=-9.228 \pm 0.209, \\
g_{K \Sigma N}^{G T} & =\frac{g_{A}^{\Sigma}}{f_{K}} \frac{\left(m_{\Sigma}+m_{n}\right)}{2}=3.215 \pm 0.163 .
\end{aligned}
$$

Using these results and the commonly cited [11] $\pi N$ coupling value $g_{\pi N N}=13.02 \pm 0.08$, it is interesting to make a simple Taylor series extrapolation for the hyperon couplings

$$
g_{K Y N}^{T S}=g_{K Y N}(0)+m_{K}^{2} \frac{d g_{K Y N}}{d q^{2}}\left(m_{K}^{2}\right) .
$$

Then assuming that the magnitudes of the GT coupling are lower bounds, as suggested from results in section III, and using the same momentum variation (derivative) as exhibited by the $\pi N$ coupling, the predicted Taylor series coupling constants are

$$
\begin{aligned}
\left|g_{K Y N}^{T S}\right| & =\left|g_{K Y N}^{G T}\right|+m_{K}^{2} \frac{g_{\pi N N}-g_{\pi N N}^{G T}}{m_{\pi}^{2}} \\
& =\left|g_{K Y N}^{G T}\right|+0.123 \frac{m_{K}^{2}}{m_{\pi}^{2}}=\left|g_{K Y N}^{G T}\right|+1.539
\end{aligned}
$$

This yields $g_{K \Lambda N}^{T S}=-10.77$ and $g_{K \Sigma N}^{T S}=4.75$ which will also be assessed in section III along with the published hyperon couplings.

TABLE I: Hadron masses, axial charges and decay constants. Errors are not listed for the very accurately known masses.

\begin{tabular}{l|r|c|c}
\hline \hline$m_{p}$ & $938.272 \mathrm{MeV}$ & & \\
$m_{n}$ & $939.565 \mathrm{MeV}$ & $g_{A}^{N}$ & $1.2695 \pm 0.0029$ \\
$m_{\Lambda}$ & $1115.683 \mathrm{MeV}$ & $g_{A}^{\Lambda}$ & $-0.718 \pm 0.015$ \\
$m_{\Sigma^{-}}$ & $1197.449 \mathrm{MeV}$ & $g_{A}^{\Sigma}$ & $0.340 \pm 0.017$ \\
$m_{\pi}$ & $139.570 \mathrm{MeV}$ & $f_{\pi}$ & $92.42 \pm .26 \mathrm{MeV}$ \\
$m_{K}$ & $493.677 \mathrm{MeV}$ & $f_{K}$ & $113.0 \pm 1.0 \mathrm{MeV}$ \\
\hline \hline
\end{tabular}

\section{B. Dashen-Weinstein sum rule}

As stated above, the GT relation is exact in the combined chiral and zero momentum limits. The deviation of $g_{M B B^{\prime}}^{G T}$ from the "physical" ${ }^{\prime}, g_{M B B^{\prime}}\left(m_{M}^{2}\right)$, defines the Goldberger-Treiman discrepancy (GTD)

$$
\Delta_{B} \equiv 1-\frac{g_{M B B^{\prime}}^{G T}}{g_{M B B^{\prime}}\left(m_{M}^{2}\right)} .
$$

\footnotetext{
1 There is some ambiguity as there is no single coupling constant because at least one vertex particle is always off-shell and different processes will have different particles off-shell.
}

There are several relations for $\Delta_{B}$, one of which is a sum rule first derived by Dashen and Weinstein [3]. It connects the GTD for the $\pi N$ and hyperon couplings and is given by

$$
g_{\pi N N} \Delta_{N}=\frac{1}{2} \frac{m_{\pi}^{2}}{m_{K}^{2}}\left(g_{K \Sigma N} \Delta_{\Sigma}-\sqrt{3} g_{K \Lambda N} \Delta_{\Lambda}\right) .
$$

While this relation is an approximation, its validity appears to be widely accepted [3, 4, 5, 6, 7] and, as detailed in Ref. [4], corrections are suppressed by two powers in the heavy baryon chiral perturbation theory expansion.

From Eq. (13) and the $\pi N$ coupling $g_{\pi N N}=13.02$, the nucleon discrepancy is $\Delta_{N}=.0094$ indicating that the GT relation is now satisfied to better than $1 \%$. Because $\Delta_{N}$ is much better known than $\Delta_{Y=\Lambda, \Sigma}$, we regard the DW sum rule as an equation between $\Delta_{\Lambda}$ and $\Delta_{\Sigma}$, which permits extracting one of the coupling constants if the other is known. Unfortunately neither is that accurately known so the sum rule only provides a correlated constraint and this is the basis of our hyperon coupling criterion. Rearranging Eq. (19) gives a linear constraint relation between $g_{K \Lambda N}$ and $g_{K \Sigma N}$

$$
g_{K \Lambda N}=\frac{1}{\sqrt{3}} g_{K \Sigma N}+b
$$

where the intercept $b$ is given by

$$
b=g_{K \Lambda N}^{G T}-\frac{g_{K \Sigma N}^{G T}}{\sqrt{3}}+\frac{2}{\sqrt{3}} \frac{m_{K}^{2}}{m_{\pi}^{2}}\left[g_{\pi N N}^{G T}-g_{\pi N N}\right] .
$$

Because the small uncertainty in $g_{\pi N N}$ is magnified by the large meson mass ratio, the constraint only restricts an area in the $\left(g_{K \Sigma N}, g_{K \Lambda N}\right)$ plane bounded by two parallel lines with the maximum and minimum intercept values corresponding to the error in $g_{\pi N N}$. Nevertheless, it still provides a new criterion for evaluating the two $g_{K Y N}$ couplings, especially when they are analyzed in tandem. This coupling constraint is applied to the published phenomenological and theoretical $g_{K Y N}$ in section III.

\section{SU(3) relation between coupling constants}

Unbroken $S U_{F}(3)$ flavor symmetry provides another relation between the baryon-meson coupling constants [12]. Using de Swart's convention, the predictions for the hyperon couplings are

$$
\begin{aligned}
& g_{K \Lambda N}^{S U(3)}=-\frac{g_{\pi N N}}{\sqrt{3}}\left(3-2 \alpha_{D}\right), \\
& g_{K \Sigma N}^{S U(3)}=g_{\pi N N}\left(2 \alpha_{D}-1\right),
\end{aligned}
$$

where $\alpha_{D}=D /(D+F)$ is the standard fraction involving $D$ and $F$-couplings. Using the $S U(6)$ value, $\alpha_{D}=0.6$, and $g_{\pi N N}=13.02$, the predicted $S U_{F}(3)$ hyperon coupling constants are $g_{K \Lambda N}^{S U(3)}=-13.53$ and $g_{K \Sigma N}^{S U(3)}=2.60$. However, flavor symmetry is broken, 
typically quoted at least 20\% [13], and we prefer using the experimental value $\alpha_{D}=0.644$ determined by Donoghue-Holstein [14]. This yields the couplings, $g_{K \Lambda N}^{S U(3)}=-12.87$ and $g_{K \Lambda N}^{S U(3)}=3.75$, and broken symmetry ranges, $-15.44 \leq g_{K \Lambda N}^{S U(3)} \leq-10.30$ and $3.00 \leq$ $g_{K \Sigma N}^{S U(3)} \leq 4.50$

We conclude this section by noting that there appears to be an inconsistency in the literature regarding phases in the DW sum rule, especially the relative sign between the nucleon and hyperon discrepancies. To ensure that Eq. (19) has the appropriate phases, we use the $S U_{F}(3)$ relations, Eqs. (22) and (23). Although the sum rule does not respect flavor symmetry, its derivation utilizes the $S U_{F}(3)$ representation for the current operator and thus the relative signs between the GT discrepancies must be the same as given in the $S U_{F}(3)$ limit. Then eliminating $\alpha_{D}$ from Eqs. 222 23) yields

$$
-\frac{2}{\sqrt{3}} g_{\pi N N} \cdot g_{K \Lambda N}^{S U(3)}=\frac{g_{K \Sigma(3)}^{S U(3)}-2 g_{\pi N N}}{\sqrt{3}} .
$$

Combining this result with the $S U_{F}(3)$ limit $\left(m_{K} \rightarrow m_{\pi}\right)$ of Eqs. (20) and (21) gives for the GT couplings

$$
\left(g_{K \Lambda N}^{G T}\right)_{S U(3)}=\frac{\left(g_{K \Sigma N}^{G T}\right)_{S U(3)}-2\left(g_{\pi N N}^{G T}\right)_{S U(3)}}{\sqrt{3}}
$$

which has the same form (and signs) as Eq. (24). Substituting the GT couplings from Eqs. [13, 14, 15 and taking the $S U_{F}(3)$ limit $\left(f_{\pi}=f_{K}, m_{B}=m_{B^{\prime}}\right)$, produces

$$
\left(g_{A}^{\Lambda}\right)_{S U(3)}=\frac{\left(g_{A}^{\Sigma}\right)_{S U(3)}-2\left(g_{A}^{N}\right)_{S U(3)}}{\sqrt{6}}
$$

Finally, inserting the $S U_{F}(3)$ axial charges, $\left(g_{A}^{N}\right)_{S U(3)}=$ $D+F,\left(g_{A}^{\Lambda}\right)_{S U(3)}=-(D+3 F) / \sqrt{6}$ and $\left(g_{A}^{\Sigma}\right)_{S U(3)}=$ $D-F$, Eq. (26) reduces to an identity verifying the phases and coefficients in our sum rule are consistent.

\section{APPLICATION OF THE CRITERION}

In the past decade there have been numerous analyses of hyperon reactions, most involving electromagnetic processes. Table II summarizes a large class of phenomenological couplings in rationalized, $g_{K Y N} / \sqrt{4 \pi}$, form. Several theoretical predictions are also included for comparison. The results of Gobbi et al. 15 are based on a Skyrme-type model, while Choe et al. 16] use a QCD sum rule method and Jeong [17] et al. employ the chiral bag model. The broken $S U_{F}(3)$ and GT values are presented along with the Taylor series extrapolation.

In Figs. 2 and 3 we compare all tabulated $\Lambda$ and $\Sigma$ couplings, respectively, in histogram form and also indicate the broken $S U_{F}(3)$ interval (dark gray), GT (diamond)

\begin{tabular}{|c|c|c|}
\hline Reference & $g_{K \Lambda N} / \sqrt{4 \pi}$ & $g_{K \Sigma N} / \sqrt{4 \pi}$ \\
\hline \multicolumn{3}{|c|}{ 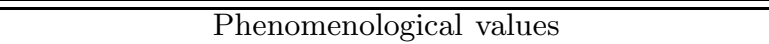 } \\
\hline Adelseck et al. [18] & -4.17 & 1.18 \\
\hline Williams et al. [19] & -1.16 & 0.09 \\
\hline \begin{tabular}{|l|} 
Williams et al. [20] \\
\end{tabular} & -2.38 & 0.27 \\
\hline Mart et al. [21] & -1.84 & 2.72 \\
\hline \begin{tabular}{|l} 
Mart et al. [21] \\
\end{tabular} & -0.84 & 1.30 \\
\hline \begin{tabular}{|l|} 
Mart et al. [21] \\
\end{tabular} & 0.51 & 0.13 \\
\hline David et al. [22] & -3.16 & 0.91 \\
\hline David et al. [22] & -3.23 & 0.80 \\
\hline \begin{tabular}{|l} 
Bennhold et al. [23] \\
\end{tabular} & -3.09 & 1.23 \\
\hline Guidal et al. $[24]$ & -3.25 & 1.26 \\
\hline Feuster et al. [25] & -2.44 to -1.73 & \\
\hline Lee et al. [26] & -3.80 & 1.20 \\
\hline Hsiao et al. [27] & -2.41 to -1.24 & -0.50 to 1.04 \\
\hline Chiang et al. [28] & -2.38 & 0.40 \\
\hline \begin{tabular}{|l|l} 
Janssen et al. [29] \\
\end{tabular} & -0.4 & \\
\hline \begin{tabular}{|l|l} 
Janssen et al. [30] \\
\end{tabular} & -0.23 & 0.28 \\
\hline Martin [31] & $-3.73^{\dagger}$ & $1.82^{\dagger}$ \\
\hline Antolin [32] & $-3.53^{\dagger}$ & $1.53^{\dagger}$ \\
\hline Timmermans et al. [33] & -3.86 & 1.10 \\
\hline \multicolumn{3}{|c|}{ Theoretical values } \\
\hline Gobbi et al. [15] & -1.06 & 0.37 \\
\hline Gobbi et al. [15] & -2.17 & 0.76 \\
\hline \begin{tabular}{|l} 
Choe et al. [16] \\
\end{tabular} & -1.96 & 0.33 \\
\hline \begin{tabular}{|l} 
Jeong et al. [17] \\
\end{tabular} & -3.77 & 1.19 \\
\hline$S U_{F}(3) \alpha_{D}=.644$ & -3.63 & 1.06 \\
\hline$S U_{F}(3)$ broken at $20 \%$ & -4.4 to -2.9 & 0.8 to 1.3 \\
\hline GT & -2.60 & 0.91 \\
\hline Taylor series & -3.04 & 1.34 \\
\hline
\end{tabular}
and Taylor series (inverted triangle) couplings.
TABLE II: Hyperon coupling constants.

${ }^{\dagger}$ Signs undetermined but taken the same as $S U_{F}(3)$.

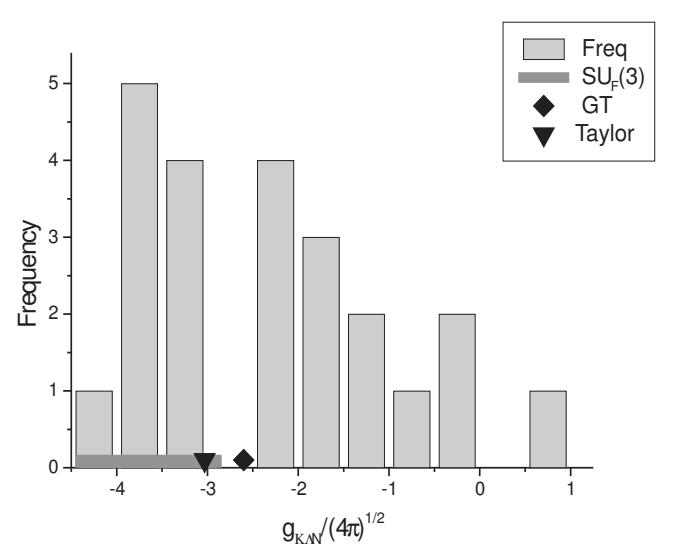

FIG. 2: GT, $S U_{F}(3)$, Taylor series and published $K \Lambda N$ coupling constants.

Applying the constraint criterion, the intercept $b$ requires specifying $q_{\pi N N}$ which is still subject to discussion 11, 34, 35, 36, 37, 38, 39]. Nevertheless, it is generally accepted that $12.90 \leq g_{\pi N N} \leq 13.20$. This uncertainty is the dominant contribution to the variation in the in- 


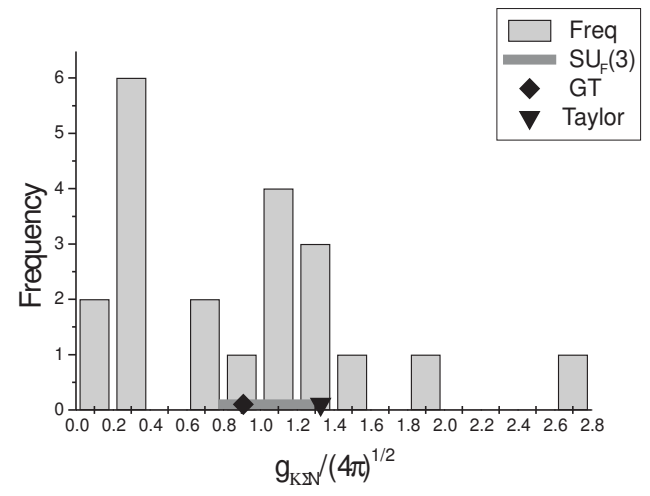

FIG. 3: GT, $S U_{F}(3)$, Taylor series and published $K \Sigma N$ coupling constants.

tercept, $\Delta b$, given by

$$
\Delta b=\sqrt{\sum_{i}\left(\frac{\partial b}{\partial x_{i}} \Delta x_{i}\right)^{2}},
$$

where the independent variables $x_{i}$ and the corresponding errors $\Delta x_{i}$ are the $\pi N$ coupling, the three axial charges $g_{A}^{B}$ and the two meson decay constants $f_{M}$. Evaluating yields $b=-13.293$ and $\Delta b=2.281$ which produces the maximum and minimum intercepts, $b_{\max } / \sqrt{4 \pi}=-11.011 / \sqrt{4 \pi}=-3.106$ and $b_{\min } / \sqrt{4 \pi}=$ $-15.575 / \sqrt{4 \pi}=-4.394$. The corresponding constraint lines are shown in Fig. 4. A more stringent constraint can be obtained from the value recommended by de Swart et al. 11], $g_{\pi N N}=13.02 \pm .08$, which produces the rationalized intercepts -3.245 and -4.011 , represented by the narrower band (dashed lines) in the figure.

Treating the coupling constants as coordinates, $\left(g_{K \Sigma N}, g_{K \Lambda N}\right)$, the values in Table II are plotted in Fig. 4 (dark circles for phenomenological, triangles for theoretical, box for $S U_{F}(3)$, diamond for GT and inverted triangle for Taylor series). The couplings satisfying the constraint are those with coordinates within the band. This produces the reduced or filtered set of acceptable values listed in Table III. The histograms for this subset of $\Lambda$ and $\Sigma$ couplings are depicted in Figs. 5 and 6 , respectively. $\Lambda$ and

Because the criterion only applies to hyperon coupling pairs $\left(g_{K \Sigma N}, g_{K \Lambda N}\right)$, two of the $\Lambda$ analyses [25, 29] were precluded from Table III. This does not necessarily mean that their results are unacceptable. Also note from Fig. 4 that not all of the broken $S U_{F}(3)$ values are inside the constraint region and if the more stringent constraint (dashed lines) is imposed, the $S U_{F}(3)$ centroid point would be eliminated from Table III along with the GT couplings. Clearly, determining a more precise $g_{\pi N N}$ will be helpful in obtaining accurate hyperon couplings.

The hyperon couplings consistent with the constraint fall in the ranges $0.80 \leq g_{K \Sigma N} / \sqrt{4 \pi} \leq 2.72$ and $-3.90 \leq$ $g_{K \Lambda N} / \sqrt{4 \pi} \leq-1.84$. The -3.90 limit on the $\Lambda$ coupling was obtained from the intersection of the constraint

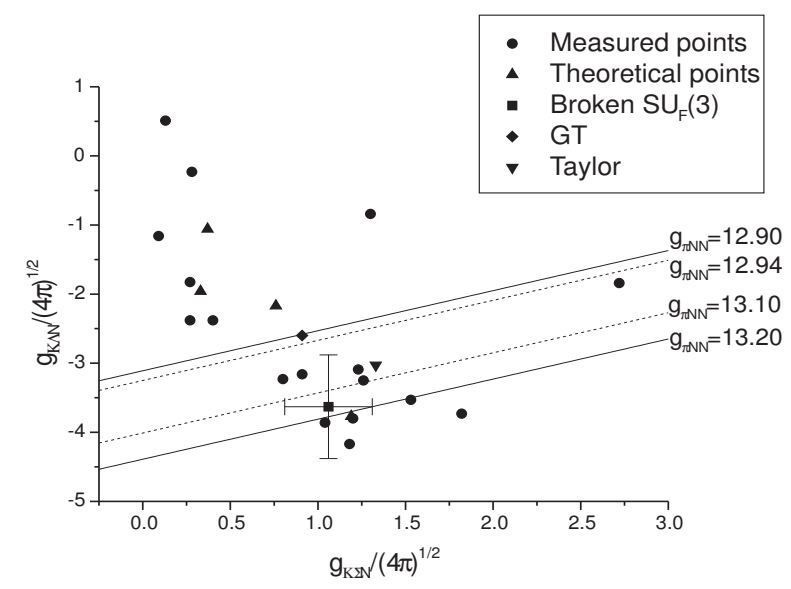

FIG. 4: Hyperon coupling coordinates and the constraint.

TABLE III: Hyperon couplings consistent with the constraint.

\begin{tabular}{||l|l|l||}
\hline \hline Reference & $g_{K \Lambda N} / \sqrt{4 \pi}$ & $g_{K \Sigma N} / \sqrt{4 \pi}$ \\
\hline \hline \multicolumn{3}{|c||}{ Phenomenological values } \\
\hline Mart et al. [21] & -1.84 & 2.72 \\
\hline David et al. [22] & -3.16 & 0.91 \\
\hline David et al. [22] & -3.23 & 0.80 \\
\hline Bennhold et al. [23] & -3.09 & 1.23 \\
\hline Guidal et al. [24] & -3.25 & 1.26 \\
\hline \multicolumn{3}{|c||}{ Theoretical values } \\
\hline$S U_{F}(3) \alpha_{D}=.644$ & -3.63 & 1.06 \\
\hline GT & -2.60 & 0.91 \\
\hline Taylor series & -3.04 & 1.34 \\
\hline \hline
\end{tabular}

line with the area representing the broken $S U_{F}(3)$ uncertainty region. Although we summarize our criterion analysis by quoting these ranges, it is important to stress that the constraint does not specify upper or lower bounds for the coupling constants. However, examining the filtered points it is interesting that, with the exception of one $\Lambda$ and one $\Sigma$ value, all couplings have magnitudes above the GT predictions. This suggests that the GT values may be lower bounds, $\left|g_{K Y N}\right| \geq\left|g_{K Y N}^{G T}\right|$, similar to the $\pi N$ coupling result, $g_{\pi N N} \geq g_{\pi N N}^{G T}$. If this proves true then the Taylor series extrapolations may be good estimates of the hyperon coupling constants.

\section{CONCLUSION}

In summary, a hyperon coupling criterion has been developed using the fundamental Goldberger-Treiman relation and the Dashen-Weinstein sum rule. Because the $\pi N$ coupling constant is not exactly known, the criterion can only restrict an area in the $\left(g_{K \Sigma N}, g_{K \Lambda N}\right)$ plane. As the precision of $g_{\pi N N}$ improves, this area will decrease providing a stronger constraint. Since the criterion can only be applied to hyperon couplings in pairs, 


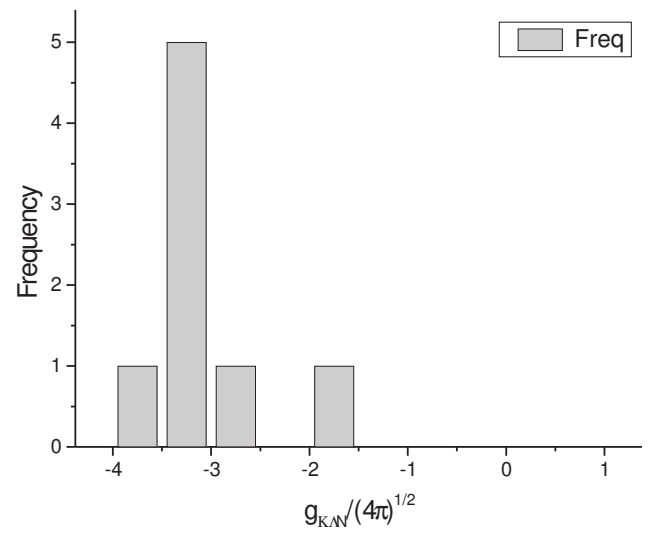

FIG. 5: Filtered $K \Lambda N$ coupling constants.

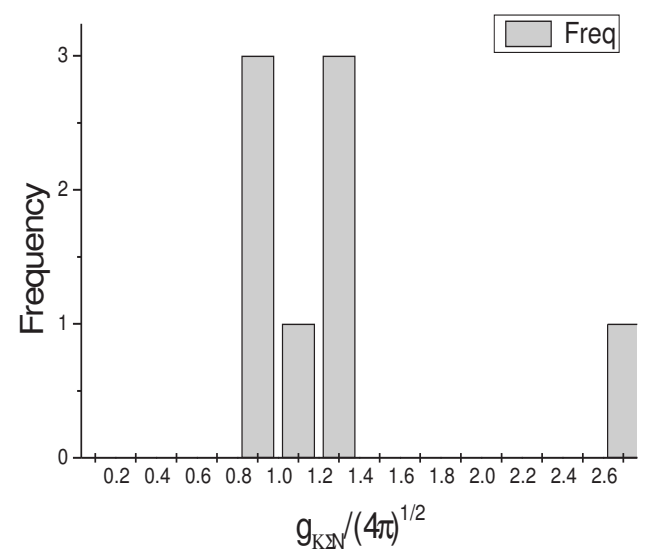

FIG. 6: Filtered $K \Sigma N$ coupling constants. phenomenological investigations incorporating this constraint should also perform a combined $\Lambda$ and $\Sigma$ data analysis, especially since the couplings are interrelated in most models. Even if there is model dependence producing renormalized, effective hyperon couplings, the constraint should still be applicable to all hyperon reactions.

This work has also applied the criterion to a large class of published couplings to produce a reduced number of $g_{K Y N}$ parameters satisfying the constraint. This subset spans the intervals $0.80 \leq g_{K \Sigma N} / \sqrt{4 \pi} \leq 2.72$ and $-3.90 \leq g_{K \Lambda N} / \sqrt{4 \pi} \leq-1.84$ and includes the $S U_{F}(3)$, GT and extrapolated Taylor series couplings. The range of absolute values in the filtered sets suggests that the GT coupling constants are, in magnitude, lower bounds. Although further study is necessary to rigorously demonstrate this, the constraint as a general guideline should be reasonably useful provided the Dashen-Weinstein sum rule is quantitatively valid.

\section{Acknowledgments}

The authors are grateful to T. Hare for informative comments. This work was supported by the Department of Energy under grant DE-FG02-97ER41048.
[1] T. Nakano et al., LEPS Collaboration, Phys. Rev. Lett. 91, 012002 (2003).

[2] M.L. Goldberger and S.B. Treiman, Phys. Rev. 110, 1178 (1958).

[3] R. Dashen and M. Weinstein, Phys. Rev. 188, 2330 (1969).

[4] J.L. Goity et al., Phys. Lett. B 454, 115 (1999).

[5] C.A. Dominguez, Riv. Nuovo Cim. 8, 1 (1985).

[6] B. Holstein, Few Body Syst. Suppl. 11, 116 (1999).

[7] N.H. Fuchs, H. Sazdjian and J. Stern, Phys. Lett. B 238, 380 (1990).

[8] CLAS Collaboration, J.W.C. McNabb et al., nucl-ex/0305028

[9] P. Bicudo and J. Ribeiro, Phys. Rev. C 55, 834 (1997).

[10] K. Hagiwara et al., Phys. Rev. D 66, 010001 (2002) and 2003 update available on the PDG WWW pages.

[11] J.J. de Swart, M.C.M. Rentmeester and R.G.E. Timmermans, PiN Newslett. 13, 96 (1997), nucl-th/9802084

[12] J.J. de Swart, Rev. Mod. Phys 35, 916 (1963).

[13] M.D. Scadron, Advanced Quantum Theory and its Applications through Feynman Diagrams (Springer-Verlag, 1991), p. 274.

[14] J.F. Donoghue and B.R. Holstein, Phys. Rev. D 25, 2015
(1982).

[15] C. Gobbi, D.O. Riska and N.N. Scoccola, Nucl. Phys. A544, 671 (1992).

[16] S. Choe, M.K. Cheoun and S.H. Lee, Phys. Rev. C 53, 1363 (1996).

[17] M.T. Jeong and Il-T. Cheon, in Proceedings of the APCTP Workshop (SNP'99), edited by IT. Cheon, S. W. Hong, and T. Motoba (World Scientific, Singapore, 2000), p. 220.

[18] R.A. Adelseck and B. Saghai, Phys. Rev. C 42, 108 (1990).

[19] R.A. Williams, C.R. Ji and S.R. Cotanch, Phys. Rev. C 43, 452 (1991).

[20] R.A. Williams, C.R. Ji and S.R. Cotanch, Phys. Rev. C 46, 1617 (1992).

[21] T. Mart, C. Bennhold and C.E. Hyde-Wright, Phys. Rev. C 51, R1074 (1995).

[22] J. C. David, C. Fayard, G.H. Lamot and B. Saghai, Phys. Rev. C 53, 2613 (1996).

[23] C. Bennhold, T. Mart and D. Kusno, in Proceedings of the CEBAF/INT Workshop on $N^{*}$ Physics, edited by TSH Lee and W. Roberts (World Scientific, Singapore, 1997), p. 166. 
[24] M. Guidal, J.M. Laget and M. Vanderhaeghen, Nucl. Phys. A627, 645 (1997).

[25] T. Feuster and U. Mosel, Phys. Rev. C 59, 460 (1999).

[26] F.X. Lee, T. Mart, C. Bennhold, H. Haberzettl and L.E. Wright, Nucl. Phys. A695, 237 (2001).

[27] S.S. Hsiao, D.H. Lu and S.N. Yang, Phys. Rev. C 61, 068201 (2000).

[28] W.-T. Chiang, F. Tabakin, T.-S. H. Lee and B. Saghai, Phys. Lett. B 517, 101 (2001).

[29] S. Janssen, J. Ryckebusch, D. Debruyne and T. Van Cauteren, Phys. Rev. C 65, 015201 (2002).

[30] S. Janssen, J. Ryckebusch, D. Debruyne and T. Van Cauteren, Phys. Rev. C 66, 035202 (2002).

[31] A.D. Martin, Nucl. Phys. B179, 33 (1981).
[32] J. Antolin, Z. Phys. C 31, 417 (1986).

[33] R.G.E. Timmermans, T.A. Rijken and J.J. de Swart, Phys. Lett. B 257, 227 (1991); Nucl. Phys. A585, 143c (1995).

[34] R. Machleidt and M.K. Banerjee, Few-Body Syst. 0, 1 (2003).

[35] M.M. Pavan et al., Phys. Scripta T87, 65 (2000).

[36] T.E.O. Ericson, B. Loiseau and A.W. Thomas, Phys. Rev. C 66, 014005 (2002).

[37] R.A. Arndt et al., Phys. Rev. C 52, 2246 (1995).

[38] T.E.O. Ericson and B. Loiseau, Phys. Lett. B 393, 167 (1997).

[39] T.E.O. Ericson et al., Phys. Rev. Lett. 75, 1046 (1995). 